\title{
An efficient algorithm for building a distributional thesaurus (and other Sketch Engine developments)
}

\author{
Pavel Rychlý \\ Masaryk University \\ Brno, Czech Republic \\ paryefi.muni.cz
}

\author{
Adam Kilgarriff \\ Lexical Computing Ltd \\ Brighton, UK \\ adame lexmasterclass.com
}

\begin{abstract}
Gorman and Curran (2006) argue that thesaurus generation for billion+-word corpora is problematic as the full computation takes many days. We present an algorithm with which the computation takes under two hours. We have created, and made publicly available, thesauruses based on large corpora for (at time of writing) seven major world languages. The development is implemented in the Sketch Engine (Kilgarriff et al., 2004).

Another innovative development in the same tool is the presentation of the grammatical behaviour of a word against the background of how all other words of the same word class behave. Thus, the English noun constraint occurs $75 \%$ in the plural. Is this a salient lexical fact? To form a judgement, we need to know the distribution for all nouns. We use histograms to present the distribution in a way that is easy to grasp.
\end{abstract}

\section{Thesaurus creation}

Over the last ten years, interest has been growing in distributional thesauruses (hereafter simply 'thesauruses'). Following initial work by (Spärck Jones, 1964) and (Grefenstette, 1994), an early, online distributional thesaurus presented in (Lin, 1998) has been widely used and cited, and numerous authors since have explored thesaurus properties and parameters: see survey component of (Weeds and Weir, 2005).
A thesaurus is created by

- taking a corpus

- identifying contexts for each word

- identifying which words share contexts.

For each word, the words that share most contexts (according to some statistic which also takes account of their frequency) are its nearest neighbours.

Thesauruses generally improve in accuracy with corpus size. The larger the corpus, the more clearly the signal (of similar words) will be distinguished from the noise (of words that just happen to share a few contexts). Lin's was based on around 300M words and (Curran, 2004) used 2B (billion).

A direct approach to thesaurus computation looks at each word and compares it with each other word, checking all contexts to see if they are shared. Thus, complexity is $O\left(n^{2} m\right)$ where $n$ in the number of types and $m$ is the size of the context vector. The number of types increases with the corpus size, and (Ravichandran et al., 2005) propose heuristics for thesaurus building without undertaking the complete calculation. The line of reasoning is explored further by (Gorman and Curran, 2006), who argue that the complete calculation is not realistic given large corpora. They estimate that, given a $2 \mathrm{~B}$ corpus and its 184,494-word vocabulary comprising all words occurring over five times, the full calculation will take nearly 300 days. With the vocabulary limited to the 75,800 words occuring over 100 times, the calculation took 18 days.

The naive algorithm has complexity $O\left(n^{2} m\right)$ but this is not the complexity of the problem. Most of 
the $n^{2}$ word pairs have nothing in common so there is no reason to check them. We proceed by working only with those word pairs that do have something in common. This allows us to create thesauruses from $1 \mathrm{~B}$ corpora in under 2 hours.

\subsection{Algorithm}

We prepare the corpus by lemmatizing and then shallow parsing to identify grammatical relation instances with the form $\left\langle w_{1}, r, w^{\prime}\right\rangle$, where $r$ is a grammatical relation, $w_{1}$ and $w^{\prime}$ are words. We count the frequency of each triple and sort all $\left\langle w_{1}, r, w^{\prime}\right.$, score $\rangle$ 4-tuples by 'contexts' where a context is a $\left\langle r, w^{\prime}\right\rangle$ pair. Only 4-tuples with positive score are included.

The algorithm then loops over each context (CONTEXTS is the set of all contexts):

\section{for $\left\langle r, w^{\prime}\right\rangle$ in CONTEXTS: \\ WLIST $=$ set of all $w$ where $\left\langle w, r, w^{\prime}\right\rangle$ exists \\ for $w_{1}$ in WLIST: \\ for $w_{2}$ in WLIST:

$$
\operatorname{sim}\left(w_{1}, w_{2}\right)+=f(\text { frequencies })^{1}
$$

The outer loop is linear in the number of contexts. The inner loop is quadratic in the number of words in WLIST, that is, the number of words sharing a particular context $\left\langle r, w^{\prime}\right\rangle$. This list is usually small (less than 1000), so the quadratic complexity is manageable.

We use a heuristic at this point. If WLIST has more than 10,000 members, the context is skipped. Any such general context is very unlikely to make a substantial difference to the similarity score, since similarity scores are weighted according to how specific they are. The computational work avoided can be substantial.

The next issue is how to store the whole $\operatorname{sim}\left(w_{1}, w_{2}\right)$ matrix. Most of the values are very small or zero. These values are not stored in the final thesaurus but they are needed during the computation. A strategy for this problem is to generate, sort and sum in sequential scan. That means that instead of incrementing the $\operatorname{sim}\left(w_{1}, w_{2}\right)$ score as we go along, we produce $\left\langle w_{1}, w_{2}, x\right\rangle$ triples in a very long list, running, for a billion-word corpus,

\footnotetext{
${ }^{1}$ In this paper we do not discuss the nature of this function as it is does not impact on the complexity. It is explored extensively in (Curran, 2004; Weeds and Weir, 2005).
}

into hundreds of GB. For such huge data, a variant of TPMMS (Two Phase Multi-way Merge Sort) is used. First we fill the whole available memory with a part of the data, sort in memory (summing where we have multiple instances of the same $\left\langle w_{1}, w_{2}\right\rangle$ as we proceed) and output the sorted stream. Then we merge sorted streams, again summing as we proceed.

Another technique we use is partitioning. The outer loop of the algorithm is fast and can be run several times with a limit on which words to process and output. For example, the first run processes only word pairs $\left\langle w_{1}, w_{2}\right\rangle$ where the ID of $w_{1}$ is between 0 and 99, the next, where it is between 100 and 199, etc. In such limited runs there is a high probability that most of the summing is done in memory. We establish a good partitioning with a dry run in which a plan is computed such that all runs produce approximately the number of items which can be sorted and summed in memory.

\subsection{Experiments}

We experimented with the $100 \mathrm{M}$-word $\mathrm{BNC}^{2}, 1 \mathrm{~B}-$ word Oxford English Corpus ${ }^{3}$ (OEC), and 1.9Bword Itwac (Baroni and Kilgarriff, 2006).

All experiments were carried out on a machine with AMD Opteron quad-processor. The machine has 32 GB of RAM but each process used only 1GB (and changing this limit produced no significant speedup). Data files were on a Promise disk array running Disk RAID5.

Parameters for the computation include:

- hits threshold MIN: only words entering into a number of triples greater than MIN will have thesaurus entries, or will be candidates for being in other words' thesaurus entries. (Note that words not passing this threshold can still be in contexts, so may contribute to the similarity of two other words: cf Daelemans et al.'s title (1999).)

- the number of words (WDS) above the threshold

\footnotetext{
${ }^{2}$ http://www.natcorp.ox.ac.uk

${ }^{3}$ http://www.askoxford.com/oec/ We are grateful to Oxford University Press for permission to use the OEC.
} 


\begin{tabular}{|l|r|r|r|r|r|}
\hline Corp & MIN & WDS & TYP & CTX & TIME \\
\hline BNC & 1 & $152 \mathrm{k}$ & $5.7 \mathrm{~m}$ & $608 \mathrm{k}$ & $13 \mathrm{~m} \mathrm{9s}$ \\
BNC & 20 & $68 \mathrm{k}$ & $5.6 \mathrm{~m}$ & $588 \mathrm{k}$ & $9 \mathrm{~m} \mathrm{30s}$ \\
OEC & 2 & $269 \mathrm{k}$ & $27.5 \mathrm{~m}$ & $994 \mathrm{k}$ & $1 \mathrm{hr} 40 \mathrm{~m}$ \\
OEC & 20 & $128 \mathrm{k}$ & $27.3 \mathrm{~m}$ & $981 \mathrm{k}$ & $1 \mathrm{hr} 27 \mathrm{~m}$ \\
OEC & 200 & $48 \mathrm{k}$ & $26.7 \mathrm{~m}$ & $965 \mathrm{k}$ & $1 \mathrm{hr} 10 \mathrm{~m}$ \\
Itwac & 20 & $137 \mathrm{k}$ & $24.8 \mathrm{~m}$ & $1.1 \mathrm{~m}$ & $1 \mathrm{hr} 16 \mathrm{~m}$ \\
\hline
\end{tabular}

Table 1: Thesaurus creation jobs and timings

- the number of triples (types) that these words occur in (TYP)

- the number of contexts (types) that these words occur in (CTX)

We have made a number of runs with different values of MIN for BNC, OEC and Itwac and present details for some representative ones in Table 1.

For the BNC, the number of partitions that the TPMMS process was divided into was usually between ten and twenty; for the OEC and ITwac it was around 200.

For the OEC, the heuristic came into play and, in a typical run, 25 high-frequency, low-salience contexts did not play a role in the theasurus computation. They included: modifier-more; modifiernot; object-of_have; subject-of-have. In Gorman and Curran, increases in speed were made at substantial cost to accuracy. Here, data from these highfrequency contexts makes negligible impact on thesaurus entries.

\subsection{Available thesauruses}

Thesauruses of the kind described are publicly available on the Sketch Engine server (http://www.sketchengine.co.uk) based on corpora of between 50M and 2B words for, at time of writing, Chinese, English, French, Italian, Japanese, Portuguese, Slovene and Spanish.

\section{Histograms for presenting statistical facts about a word's grammar}

$75 \%$ of the occurrences of the English noun constraint in the BNC are in the plural. Many dictionaries note that some nouns are usually plural: the question here is, how salient is the fact about con-

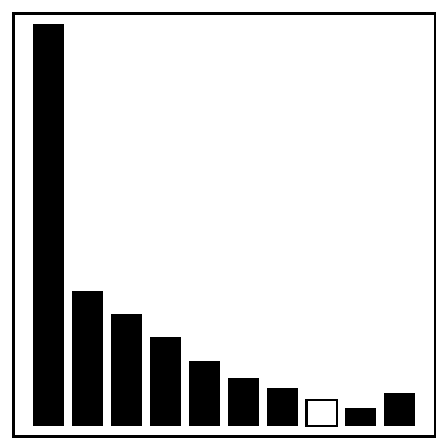

Figure 1: Distribution of nouns with respect to proportion of instances in plural, from 0 to 1 in 10 steps, with the class that constraint is in, in white.

\section{straint $?^{45}$}

To address it we need to know not only the proportion for constraint but also the proportion for nouns in general. If the average, across nouns, is $50 \%$ then it is probably not noteworthy. But if the average is $2 \%$, it is. If it is $30 \%$, we may want to ask a more specific question: for what proportion of nouns is the percentage higher than $75 \%$. We need to view " $75 \%$ plural" in the context of the whole distribution.

All the information is available. We can determine, in a large corpus such as the BNC, for each noun lemma with more than (say) fifty occurrences, what percentage is plural. We present the data in a histogram: we count the nouns for which the proportion is between 0 and $0.1,0.1$ and $0.2, \ldots, 0.9$ and 1. The histogram is shown in Fig 1, based on the 14,576 nouns with fifty or more occurrences in the BNC. (The first column corresponds to 6113 items.) We mark the category containing the item of interest, in red (white in this paper). We believe this is an intuitive and easy-to-interpret way of presenting a word's relative frequency in a particular grammatical context, against the background of how other words of the same word class behave.

We have implemented histograms like these in the Sketch Engine for a range of word classes and grammatical contexts. The histograms are integrated into

\footnotetext{
${ }^{4}$ Other $75 \%$ plural nouns which might have served as the example include: activist bean convulsion ember feminist intricacy joist mechanic relative sandbag shutter siding teabag testicle trinket tusk. The list immediately suggests a typology of usually-plural nouns, indicating how this kind of analysis provokes new questions.

${ }^{5}$ Of course plurals may be salient for one sense but not others.
} 
the word sketch ${ }^{6}$ for each word. (Up until now the information has been available but hard to interpret.) In accordance with the word sketch principle of not wasting screen space, or user time, on uninteresting facts, histograms are only presented where a word is in the top (or bottom) percentile for a grammatical pattern or construction.

Similar diagrams have been used for similar purposes by (Lieber and Baayen, 1997). This is, we believe, the first time that they have been offered as part of a corpus query tool.

\section{Text type, subcorpora and keywords}

Where a corpus has components of different text types, users often ask: "what words are distinctive of a particular text type", "what are the keywords?"? Computations of this kind often give unhelpful results because of the 'lumpiness' of word distributions: a word will often appear many times in an individual text, so statistics designed to find words which are distinctively different between text types will give high values for words which happen to be the topic of just one particular text (Church, 2000). (Hlaváčová and Rychlý, 1999) address the problem through defining "average reduced frequency" (ARF), a modified frequency count in which the count is reduced according to the extent to which occurrences of a word are bunched together.

The Sketch Engine now allows the user to prepare keyword lists for any subcorpus, either in relation to the full corpus or in relation to another subcorpus, using a statistic of the user's choosing and basing the result either on raw frequency or on ARF.

\section{Acknowledgements}

This work has been partly supported by the Academy of Sciences of Czech Republic under the project T100300419, by the Ministry of Education of Czech Republic within the Center of basic research LC536 and in the National Research Programme II project $2 \mathrm{C} 06009$.

\footnotetext{
${ }^{6} \mathrm{~A}$ word sketch is a one-page corpus-derived account of a word's grammatical and collocation behaviour.

${ }^{7}$ The well-established WordSmith corpus tool (http://www.lexically.net/wordsmith) has a keywords function which has been very widely used, see e.g., (Berber Sardinha, 2000).
}

\section{References}

Marco Baroni and Adam Kilgarriff. 2006. Large linguistically-processed web corpora for multiple languages. In $E A C L$.

Tony Berber Sardinha. 2000. Comparing corpora with wordsmith tools: how large must the reference corpus be? In Proceedings of the ACL Workshop on Comparing Corpora, pages 7-13.

Kenneth Ward Church. 2000. Empirical estimates of adaptation: The chance of two noriegas is closer to p/2 than p2. In COLING, pages 180-186.

James Curran. 2004. From Distributional to Semantic Similarity. Ph.D. thesis, Edinburgh Univesity.

Walter Daelemans, Antal van den Bosch, and Jakub Zavrel. 1999. Forgetting exceptions is harmful in language learning. Machine Learning, 34(1-3).

James Gorman and James R. Curran. 2006. Scaling distributional similarity to large corpora. In $A C L$.

Gregory Grefenstette. 1994. Explorations in Automatic Thesaurus Discovery. Kluwer.

Jaroslava Hlaváčová and Pavel Rychlý. 1999. Dispersion of words in a language corpus. In Proc. TSD (Text Speech Dialogue), pages 321-324.

Adam Kilgarriff, Pavel Rychlý, Pavel Smrž, and David Tugwell. 2004. The sketch engine. In Proc. EURALEX, pages 105-116.

Rochelle Lieber and Harald Baayen. 1997. Word frequency distributions and lexical semantics. Computers in the Humanities, 30:281-291.

Dekang Lin. 1998. Automatic retrieval and clustering of similar words. In COLING-ACL, pages 768-774.

Deepak Ravichandran, Patrick Pantel, and Eduard H. Hovy. 2005. Randomized algorithms and nlp: Using locality sensitive hash functions for high speed noun clustering. In $A C L$.

Karen Spärck Jones. 1964. Synonymy and Semantic Classificiation. Ph.D. thesis, Edinburgh University.

Julie Weeds and David J. Weir. 2005. Co-occurrence retrieval: A flexible framework for lexical distributional similarity. Computational Linguistics, 31(4):439-475. 